\title{
Germanica
}

contemporain : jeux intermédiaux, modes de transfert, adaptations

\section{Fiktion-Gesellschaft-Differenzen am Beispiel filmischer Raumkonstruktionen in deutschsprachiger Gegenwartsliteratur der 2000er Jahre}

Film-aesthetic space structures to explicit literary/social differences in german literature in the $2000 \mathrm{~s}$

Des différences sociales et littéraires illustrées par le biais de constructions d'espaces filmiques dans la littérature germanophone des années 2000

\section{Jan Drees}

\section{OpenEdition}

\section{Journals}

Édition électronique

URL : http://journals.openedition.org/germanica/2272

DOI : $10.4000 /$ germanica.2272

ISSN : 2107-0784

Éditeur

Université de Lille

Édition imprimée

Date de publication : 30 décembre 2013

Pagination : 45-60

ISBN : 9782913857322

ISSN : 0984-2632

Référence électronique

Jan Drees, «Fiktion-Gesellschaft-Differenzen am Beispiel filmischer Raumkonstruktionen in deutschsprachiger Gegenwartsliteratur der 2000er Jahre », Germanica [Online], 53 | 2013, Online erschienen am: 30 Dezember 2016, abgerufen am 06 Oktober 2020. URL : http://

journals.openedition.org/germanica/2272 ; DOI : https://doi.org/10.4000/germanica.2272 


\title{
Fiktion-Gesellschaft-Differenzen am Beispiel filmischer Raumkonstruktionen in deutschsprachiger Gegenwartsliteratur der 2000er Jahre
}

\author{
Jan DREES \\ Universität Münster
}

Gehört das System der Literatur zur Gesellschaft, oder ist es, anders als Systeme wie Recht, Politik, Medizin, Moral, von dem, was Gesellschaft genannt wird, differenziert? Vermutet wird, dass ein Teil der deutschen Gegenwartsliteratur der 2000er Jahre eben diese Fragestellung auf eine spezifische Art und Weise thematisiert, und zwar derart eindrucksvoll, dass hieraus nahezu im Verborgenen eine Strömung entstanden ist, welche die beiden zeitgleich zu beobachtenden Phänomene der so genannten „Pop-“ und der „Fräuleinliteratur“ an Wirkungsmacht übertrifft.

$\mathrm{Zu}$ belegen ist, dass Texte existieren, die auf eine spezifische Weise „filmische Räume“ inszenieren, um eben diese Literatur/ Gesellschaft-Differenz von der Realitäts- bzw. Gesellschaftsebene auf die Fiktionsebene zu überführen.

Nach einer kurzen, für den vorliegenden Aufsatz nutzbar gemachten Definition filmischer Räume folgt ein ebenso kurzer Überblick über das Problem der nach Niklas Luhmanns Systemtheorie beschreibbaren Differenz von Literatur und Gesellschaft. Anhand von drei exemplarischen Texten der in besagten 2000er Jahren veröffentlichenden Thomas 
Glavinic und Thomas von Steinaecker wird gezeigt, wie der filmische Raum als „Text im Text“ inszeniert wird. Die Argumentation meiner These steht in Bezug zu einem mittlerweile 30 Jahre zurückliegenden Happening und drei literaturwissenschaftlichen Studien jüngeren Datums ${ }^{1}$.

Beim Bachmann-Preis 1983 schnitt sich der damals 29-jährige Punkautor Rainald Goetz vor Publikum in die Stirn. Sein Blut tropfte auf das Papier. Auf diesem stand Goetz' Text Subito, der von einem Autor erzählt, der zu den Tagen der deutschen Literatur nach Klagenfurt fährt, sich dort während seiner Lesung in die Stirn ritzt - und blutet.

Die im System der Gesellschaft angelegte Leseperformance tritt dadurch in eine Interdependenz zum literarischen Text. Goetz löst die Differenz Gesellschaft/Literatur zugunsten einer „,besseren Differenz“ auf. Indem Goetz' reales Blut aufs Papier tropft, in den fiktionalen Text jedoch nicht eindringt, bleibt folgende Frage offen: Blutet der Autor, weil es im Text steht, oder steht das Blut im Text, weil sich der Autor bei der späteren Lesung schneidet? Durch eine sich nicht auszutauschende Realitätsverdopplung wird die Differenz Gesellschaft/Literatur bzw. Realität/Fiktionalität explizit² .

Dieses Verfahren behält Goetz bis heute bei. Natalie Binczek schreibt in ihrem Aufsatz über die Poetik des Sekundären in Goetz' loslabern: „Was der Ich-Erzähler hier - im Konjunktiv - erwägt und als einen ,praktischen Theoretismus' bezeichnet, deutet auf ein Werkkonzept hin, das die Differenz von Text und Kommentar bzw. Text und Epitext untergräbt, weil es beides beinhaltet und nur, wenn es beides beinhaltet, d.h. also auch sämtliche Reaktionen auf den Text antizipiert, ein ,fertiges Buch" werden kann"3.

Goetz bedient sich theatraler Topographien, um die von Luhmann behauptete Kunst/Gesellschaft-Differenz zu markieren. In der folgenden Analyse soll gezeigt werden, wie knapp 20 Jahre später neue Ausbildungen filmischer Topographien zur Markierung der Kunst/Gesellschaft-Differenz instrumentalisiert werden. In dieser Argumentation geht es keineswegs um intertextuelle Verweise, also um die Frage, welche Filme in den zu beobachtenden Texten aufscheinen ${ }^{4}$.

1. - Moritz Baßler: Der deutsche Pop-Roman: Die neuen Archivisten, München, C.H. Beck, 2002./Johanna Bohley, Julia Schöll (Hgg.): Das erste Jahrzehnt: Narrative und Poetiken des 21. Jahrhunderts, Würzburg, Königshausen und Neumann, 2011./Susanne Krones, Evi Zemanek (Hgg.): Literatur der Jahrtausendwende: Themen, Schreibverfahren und Buchmarkt um 2000, Münster, transcript, 2008.

2. - Vgl. Jan Drees: Rainald Goetz - Irre mit System. Wien, Arco, 2010.

3. - Natalie Binczek: Fernsehauftritte der Literatur: Rainald Goetz. In: Sprache und Literatur, Nr. 109, 43. Jg. 2012, 1. Hj. Paderborn, Wilhelm Fink, 2012, S. 74.

4. - Diese Frage wurde für die deutschsprachige Literatur der 2000er Jahre hinlänglich geklärt, beispielsweise von Moritz Baßlers Studie Der deutsche Pop-Roman: 


\section{Narratologien im Film um das Jahr 2000}

Die topographische Materialisierung der hier beobachteten deutschen Gegenwartsliteratur steht unter dem massiven Eindruck von sieben Kinofilmen, die zwischen 1998 und 2001 erschienen sind: In Lola rennt (1998) von Tom Tykwer erscheint die erzählte Welt als eine von vielen möglichen Welten, die Handlung ist inkonsistent, präsentierte Szenen werden stets aufs Neue zugunsten einer geänderten histoire zurückgenommen.

Fight Club (1999) präsentiert einen schizophrenen Helden, dessen zwei Persönlichkeiten, für den Zuschauer nicht dechiffrierbar, von unterschiedlichen Schauspielern (Edward Norton, Brad Pitt) dargestellt und erst zum Schluss als Realität/Wahn-Differenz markiert werden.

Im Blockbuster The Matrix (1999) erkennt der Hacker Thomas A. Anderson, dass seine Realität lediglich eine computerbasierte Virtual Reality ist. In der ,echten Wirklichkeit“" wurden die Menschen von einer kollektiv-künstlichen Intelligenz versklavt. Ihr Bewusstsein lebt in programmierten Träumen. Im Kriminalfilm Memento (2000) werden, zunächst unmarkiert, ein chronologisch fortlaufender und ein chronologisch entgegengesetzter Handlungsstrang präsentiert.

Der Mystery-Thriller Mulholland Drive (2001) operiert mit zahlreichen Handlungswechseln und Motivumdeutungen. Die Darsteller der beiden Hauptfiguren wechseln zur Filmmitte, sodass der Aufbau einer diegetischen Realität hintertrieben wird.

The Sixth Sense (1999) erzählt die Geschichte einer bereits toten Figur, deren Ermordung zu Beginn des Films gezeigt wird, ohne jedoch im Folgenden aufzuklären, dass nicht die Realität der Lebenden, sondern die Realität des Toten präsentiert wird. Dieses formalen Kunstgriffes bedient sich ebenso The Others (2001), wenn die scheinbar von Geistern heimgesuchten Figuren als die tatsächlich Untoten demaskiert werden.

Den sieben Filmen gemein ist ihre Abkehr von gewohnten filmischen Erzählverfahren. In konstruktivistischer Manier wird die mise en scène als Mimesis der Realität inszeniert, um später eine Differenz zwischen Realität und Virtualität zu markieren, und der metaphysisch schicksalhaften Notwendigkeit ein Kontingenzprinzip entgegenzusetzen.

Die im Folgenden zu analysierenden Texte Glavinics und von Steinaeckers spielen mit diesen bereits aus dem Film bekannten Realität-Virtualität-Differenzen. Vermittels einer „Beobachtung zweiter

Die neuen Archivisten aus dem Jahr 2002, ebenso in dem von Evi Zemanek und Susanne Krones herausgegebenen Band Literatur der Jahrtausendwende: Themen, Schreibverfahren und Buchmarkt um 2000 aus dem Jahr 2008, und in der Sammlung Das erste Jahrzehnt: Narrative und Poetiken des 21. Jahrhunderts, 2011 herausgegeben von Julia Schöll und Johanna Bohley. 
Ordnung“ den virtuellen Raum als Film- oder Bild-Raum in Differenz zum Text-Raum inszenieren ${ }^{5}$.

\section{Der filmische Raum}

Angelehnt an Rayd Khoulokis maßgebliche Studie über Konstruktion, Wahrnehmung und Bedeutung des filmischen Raums wird dieser formal definiert als mise en scène, bei der eine zweidimensional projizierte Aufnahme den zuvor abgefilmten dreidimensionalen Raum lediglich vorspiegelt. Die einzeln fotografierten Bilder illusionieren in der Projektion eine tatsächlich nicht existierende Bewegung:

Dieser Eindruck wird durch den ständigen Perspektivwechsel verstärkt, der mit der Bewegung einhergeht, sei es die Bewegung der Kamera oder die Bewegung vor ihr. Fährt die Kamera zum Beispiel um einen Gegenstand herum, erfährt der Zuschauer dessen dreidimensionale Ausdehnung im Bewegungsablauf ${ }^{6}$.

Khouloki stellt mittels Auswertung des filmtheoretischen Kanons - von Balázs' Der Film. Werden und Wesen einer neuen Kunst über Eisensteins Montage 1938 bis zu Robin Curtis' Über die Grenzen des Körpers hinaus, einen Überblick zusammen, der nahezu alle im Film verwendeten Gestaltungsmittel vorstellt, die bisher für die Raumillusionierung und -inszenierung nutzbar gemacht worden sind. Beschrieben wird „in einer Art phänomenologischer Darstellung“ (Khouloki S. 96), welche Möglichkeiten der Konstruktion filmischen Raums entwickelt wurden, in welcher Weise neben den bereits oben aufgezählten Techniken auch Verfahren der Raumbeleuchtung, die Verwendung von Tiefenschärfe und -unschärfe oder die Schaffung nichteuklidischer Räume die Beobachtung des Zuschauers lenken.

Ein separates Kapitel analysiert Raumdynamisierungen durch unterschiedliche Arten der Kameraführung, vergleicht Montagetechniken und erläutert, wieso der Zuschauer den ihm präsentierten Filmraum über die Grenzen der Leinwand hinaus verlängert und in welcher Weise beispielsweise die Zeitlupe in hyperrealer Manier Strukturen offenlegt, die dem Bewusstsein üblicherweise unbekannt sind. Montagen, Schnitte, Überblendungen, die Verwendung verschiedener Brennweiten

5. - „MULHOLLAND DRIVE ist ein gutes Beispiel für eine Tendenz in aktuellen, auch populären Filmen (wie etwa THE OTHERS [...] THE SIXTH SENSE [...] FIGHT CLUB), in denen die Zuverlässigkeit des Gezeigten hintertrieben wird, indem sie sich den Realitätseindruck des Filmbildes zunutze machen - und damit auch die Konvention, nach der das, was gezeigt wird, vom Zuschauer innerhalb der diegetischen Welt auch als real angenommen wird, solange es nicht entsprechend den Konventionen als Traum oder Ähnliches markiert wird." Rayd Khouloki : Der filmische Raum - Konstruktion, Wahrnehmung, Bedeutung, Berlin, Bertz + Fischer, 2009, S. 183.

6. - Khouloki: Der filmische Raum, 2009, S. 11. 
und perspektivische Bildkonstruktionen, welche wiederum zurückgreifen auf Erkenntnisse der Kognitionspsychologie, qualifizieren die Inszenierung des filmischen Raums als singuläre Kunstform, die sich insbesondere aufgrund ihrer technischen Möglichkeiten deutlich von anderen Kunstformen abgrenzt.

Zwar gibt es Rückgriffe, wie auf die in der Malerei entdeckte „Zentralperspektive“. Jedoch hat ein steter technischer Fortschritt, von der ersten Kamerafahrt bis zum neuesten Computertrick, die Möglichkeit derart spezifischer Weltkonstruktionen geschaffen, dass deren Anlehnung in einem literarischen Text deutlich auf den Film rückführbar ist. Es ist also keinesfalls so, dass ,filmische Räume“ lediglich durch Übernahmen von Kriterien anderer (sozialer, körperlicher, politisch-geographischer) Räume definiert sind.

In einem weiteren Teil seiner Studie stellt Khouloki den technischen Möglichkeiten nun ästhetische Parameter gegenüber: „Es geht nicht darum, ob ein Bild zentralperspektivisch, mit Weitwinkel- oder Teleobjektiv oder ähnlichem konstruiert ist, sondern darum, welche Raumwahrnehmung erzeugt wird. Ist ein Raum zum Beispiel beengend, oder evoziert er ein Gefühl von Weite? Betont die Raumkonstruktion die Flächigkeit des Bildes, oder versucht sie den Eindruck von Tiefe zu erzeugen?" (Khouloki, S. 213).

Bei der Analyse der hier vorgestellten Texte werden jene filmwissenschaftlichen Termini verwendet, die Khouloki in seiner Studie erstmalig bereitstellt. Mit diesen Begriffen wird gefragt, in welchem Bezug die sinnlich zugängliche „Realität“ und die dargestellte, konstruierte Fiktionalität des Films in den jeweiligen literarischen Texten stehen. Daraus wird die Problematisierung einer grundsätzlichen Differenz literarischer Beobachtung im Verhältnis zu dem was Luhmann „Gesellschaft" nennt eröffnet.

\section{Luhmanns Differenz von Kunst/Gesellschaft}

Da im Folgenden zu analysieren ist, in welcher Weise der Aufbau filmischer Räume eine System-Umwelt-Differenz explizit macht, soll kurz auf Niklas Luhmanns Verständnis der „Literatur als Beobachtung 2. Ordnung" eingegangen werden. In seinem Essay Weltkunst ${ }^{7}$ wird analysiert, in welcher Weise Kunst die Welt beobachtet. Moderne Kunst möchte selbst als Beobachter wahrgenommen werden, nicht mehr bloße Mimesis sein 8 . Durch Form konstruiert der Künstler laut Luhmann eine fiktionale Realität, die der ontologisch fassbaren realen

7. - Niklas Luhmann: Weltkunst. In: Ders.: Schriften zu Kunst und Literatur, Frankfurt. Suhrkamp, 2008.

8. - Vgl. Stefan Lüddemann: Kunstkritik als Kommunikation - Vom Richteramt zur Evaluationsagentur, Wiesbaden, DUV, 2004. 
Realität gegenübersteht. Dieser Formunterschied lässt schlussfolgern, dass die Realität der Kunst von der Realität differenziert ist.

In Luhmanns Die Kunst der Gesellschaft werden Kunstwerke des Weiteren als realisierte Beobachtungen zweiter Ordnung klassifiziert. „Das Kunstwerk stellt seine eigene Realität fest, die sich von der gängigen Realität unterscheidet" 9 . In ihr können auch nicht realisierte Möglichkeiten der realen Realität angeboten werden, wobei von Vision bis Kritik alles ausführbar wird. Teilsysteme beobachten die sie umgebende Umwelt nach einer jeweils spezifischen Leitdifferenz, sie operieren mittels eines binären Codes, der sie zunächst von ihrer Umwelt unterscheidet, mit dem sie aber auch selbst unterscheiden, innerhalb und in Bezug auf die Umwelt des Systems: „Der die Operationen des Rechts anleitende Code besteht in der binären Differenz Recht/ Unrecht" (Ebd., S. 147). Kunst beobachtet ihre Umwelt nach dem Code „schön/hässlich“10.

Luhmann rekonstruiert in Die Literatur als fiktionale Realität den 300 -jährigen Weg nicht-realer Lektüre ${ }^{11}$. Ihr frühes Anliegen soll darin bestanden haben, die Realitätsferne unmodischer Ritterromane zu umgehen, gleichzeitig jedoch eine ,virtuelle Welt“ (Ebd., S. 277) zu präsentieren, die „als andere Welt erfahren“ (Ebd. S. 277) werden kann. Durch die Figur des „,re-entry“ 12 wird die Differenz von realer und fiktionaler Realität ins System der Fiktionalität wieder eingeführt: „Mit Realität ist einerseits eine Seite der Unterscheidung, nämlich das Antonym zu Fiktionalität bezeichnet, aber andererseits auch die Unterscheidung selbst, die ja irgendwie in der Welt getroffen wird“ (Ebd., S. 281).

Da sie fiktional und eben nicht an die Kompossibilität der realen Realität gebunden ist, entsteht selbst erzeugte Kontingenz. Diese kann aufgrund ihrer „memory function“ (Ebd., S. 282). (Vergangenheit) und „oscillator function“ (Ebd., S. 282). (Zukunft) bei jeder Unterscheidung die Richtung wechseln. „Das Resultat ihrer vorherigen Operationen

9. - Claudio Baraldi/Giancarlo Corsi/Elena Esposito: GLU - Glossar zu Niklas Luhmanns Theorie sozialer Systeme, Frankfurt, Suhrkamp, 1997, S. 107.

10. - „Die Beobachtung der Kunst basiert auf einem spezifischen Code, der in der traditionellen Ästhetik mit der Unterscheidung schön/hässlich ausgedrückt wurde. Heute wird diese Unterscheidung auf die Alternative passt/passt-nicht uminterpretiert.“ Baraldi et.al., S. $105 \mathrm{ff}$.

11. - Er beginnt mit Guilleragues Lettres portugaises von 1669 und der englischen Literatur des 17. Jahrhunderts, die ebenfalls „Texte als gefundene Dokumente“ vorstellte und somit versuchte, Realität und Fiktionalität zu verbinden. Vgl. Luhmann, 2008, S. 277-278.

12. - Luhmann übernimmt hier ein mathematisches Formenkalkül von George Spencer Brown, das ,den Anspruch erhebt [...], Paradoxien auflösen zu können, die für die mathematischen Operationen der Arithmetik und der Algebra unzugänglich bleiben.“ Ebd., S. 281. 
ist ihr durch ihre memory function gegeben, ihre Zukunft wird durch ihre oscillator function strukturiert. Sie strebt nicht bestimmte Zielzustände an (etwa: immer bessere Kunstwerke zu produzieren). Fiktionalität richtet sich nicht mehr nach der Vergangenheit aus, strebt keine Mimesis oder Imitation an, sondern beobachtet mit Blick in die Zukunft (Vgl. Ebd., S. 283), als „laufende Renovierung der Zeit.“ (Ebd., S. 284). Eine Unterscheidung trennt laufend Vergangenheit (deren Erkenntnisse akzeptiert oder abgelehnt werden) und Zukunft (als Möglichkeitsraum) ${ }^{13}$.

In einer Zusammenführung von Khoulokis Annahme, dass der Zuschauer den gezeigten Raum über die Grenze des Bildes hinaus verlängert, kann bis in die späten 1990er Jahre davon ausgegangen werden, dass sich in Literatur vermittelte Filmtopographien an Rezeptionstraditionen des Mediums „Film“ orientieren. Durch neue Raumillusionierungen im Film der 2000er Jahre erhöht sich die Possibilität literarisch möglicher Filmdarstellungen. Die aktualisierte Darstellung filmischer Wirklichkeit in der Literatur bezieht sich nicht mehr auf die zuvor angenommene Konsistenz. Wenn eine Figur in der ersten Szene stirbt und in der darauf folgenden Szene lebt, so bedeutet dies nicht (mehr) automatisch, dass sie den Mordanschlag überlebt hat (The Sixth Sense). Eine im discours realisierte Szenenfolge kann sich zeitlich entgegengesetzt zur histoire aktualisieren (Memento). Figur und Darsteller können voneinander unabhängig realisiert sein. Zwei Darsteller erscheinen dann als Möglichkeitsformen einer Figur (Fight Club, Mulholland Drive). Die Diegese des Films erscheint inkonsistent (Lola rennt), als Film im Film (The Matrix), die zweite Beobachtungsebene eigentlich als die erste (The Others), usw.

\section{Thomas Glavinic: Der Kameramörder}

In seinem 2001 veröffentlichten dritten Roman Der Kameramörder entwirft der 29-jährige Wiener Schriftsteller Thomas Glavinic ein FilmRaum-Szenario, das er bei seinem fünf Jahre später erscheinenden „Meisterstück“14 Die Arbeit der Nacht formal perfektioniert. Bereits die Paratexte der Erstausgabe mit ihrem dem Teletext nachempfundenem Coverlayout zeigt an, dass hier eine über den Bildschirm vermittelte Realität dargeboten wird. Auf der vierten Umschlagseite des Buchs steht:

13. - „Was Literaturwissenschaftler ,Intertextualität“ nennen, ist nichts anderes als ein Resultat der durch die Zeit erzwungenen ständigen Neubeschreibungen. Das, was ernst gemeint war, wird jetzt parodiert oder ironisch behandelt. Was gültig war, wird so behandelt, daß es fast nicht wiedererkannt werden kann." Ebd., S. 285.

14. - Daniel Kehlmann: „Die Hölle sind nicht die anderen“. In: Der Spiegel, 31/2006. Hamburg, 2006, S. 128. 
,Jeder Mensch ist ein Abgrund', [...] und oft will es scheinen, als sei das der Stoff, aus dem das Fernsehen seine Einschaltquoten macht. Manches Verbrechen wird wegen dieser Quote begangen, auch das des Kameramörders. Er hat zwei Kinder gezwungen, sich umzubringen, und sie dabei gefilmt. Aus dem Osterfest wird eine Orgie des Hasses und der Sensationslust, aus der Erzählung von Thomas Glavinic eine bittere, sarkastische, atemberaubende Warnung, was mit uns allen schnell geschehen kann ${ }^{15}$.

Das Cover ist auf der Textebene wie eine pixelige Teletextseite gestaltet. Der Hintergrund zeigt eine Fotografie, die im Teletext nicht wiedergegeben werden kann, sondern bereits eine weitere Realitätsebene markiert: Es ist ein Blick in den Wald hinein, der im Buch wesentlich auftaucht. Beide Ebenen sind Teil des diegetischen Raums ${ }^{16}$, stehen jedoch in einem Beobachtungsverhältnis zueinander (der Bildschirm als Medium der Beobachtung einer Fahndung).

Der Kameramörder wird sowohl mit dem ersten, ohne vorangestelltem Zitat präsentierten Satz ,Ich wurde gebeten, alles aufzuschreiben“ (KM, S. 5), als auch durch genaue Ortsangaben wie ,wohnhaft in Kaibing 6, 8537 Kaibing“ (Ebd.) und zahlreiche Abkürzungen wie ,8x“ (KM, S 6.), ,u.dgl.“ (Ebd), ,,Z.Zt.“ (KM, S. 64) als Protokoll eines nicht identifizierbaren, ,etwa 30jährigen, mittelgroßen“ (KM, S. 8) Ich-Erzählers inszeniert. Dieser ist mit seiner Gattin Sonja Wagner über die Osterfeiertage beim befreundeten Ehepaar Heinrich und Eva Stubenrauch in der Weststeiermark eingeladen. Man vertreibt sich die Zeit mit ereignislosen Tischtennispartien, Einkäufen, gemeinsamen Essen, mit Gesprächen.

Ereignisreich ist dagegen die Medienberichterstattung in Zeitungen, im Fernseh-, ORF-Teletext-, Radioprogramm über einen gerade verübten Mord an zwei Kindern. Es gibt „Sondersendungen“ (KM, S. 19), „Laufzeilen am unteren Bildrand“ (Ebd.), „Programmänderungen aus aktuellem Anlaß“ (KM, S. 41), eine Ausgabe der Kronen Zeitung „mit 16 Seiten Bildbericht über den Mord“ (KM, S. 82) und Livebilder aus einem Hubschrauber (KM, S. 107 ff.) Die umfangreiche Medienrezeption wird ausführlich protokolliert, als Beobachtung einer Beobachtung, der immer dann, wenn die Kamerabilder des Mordes

15. - Thomas Glavinic: Der Kameramörder, Berlin, Volk + Welt, 2001. Nachfolgend zitiert als KM.

16. - „Ort meint einen Schauplatz, an dem sich eine Handlung abspielt und von den Teilansichten für den Zuschauer in einzelnen Einstellungen sichtbar ist. Es kann sich dabei um Gebäude, eine Straße, den Teil einer Landschaft oder anderes handeln. Die Übergänge zwischen zwei Orten können dabei fließend sein, sodass nicht immer klar zu entscheiden ist, wo ein Ort seine Grenze hat und der nächste anfängt. Der Begriff ,diegetischer Raum" bezieht sich auf die gesamte im Film dargestellte Welt mit allen sichtbaren und nichtsichtbaren Teilen."Khouloki, 2009, S. 92. 
eingespielt und durch Laufzeilen kommentiert werden, eine weitere Beobachtungsebene hinzugefügt wird.

Vergleichbar mit der von Khouloki analysierten Realitätsillusionierung in Filmen wie Fight Club oder The Sixth Sense wird jede einzelne Ebene in Der Kameramörder nach Erzählkonventionen inszeniert, die ein je getreues Abbild der Realität suggerieren, d.h.:

- Das Video des Kameramörders behauptet eine Differenz zwischen dem handelnden Mörder und dem beobachtendem Erzähler.

- Die Fernsehbilder der polizeilichen Ermittlungsbemühungen behaupten ebenfalls eine Differenz zwischen Fernsehraum und Beobachterraum.

- Die beschriebene Gemeinschaft der beiden Ehepaare, ihr privates Beisammensein an den Ostertagen wird in eine behauptete Differenz zum beruflichen Tun der Polizisten, Reporter, Nachrichtensprecher gesetzt.

Tatsächlich ist der Ich-Erzähler zugleich der Kameramörder. Auf den letzten Seiten bilden die zuvor in Differenz gesetzten Bilder der Kamera und jene des Ich-Erzählers eine Einheit: „Auf dem Bildschirm beobachtete ich, wie die neben mir mit dem Tablett stehende Eva einige Meter zurückwich.“ (KM, S. 156) Der berichtende Beobachter von Medienbeiträgen über den ebenfalls beobachtenden Kameramörder wird sowohl Subjekt als auch Objekt seiner Beobachtung, sodass in einem letzten Beobachtungsakt allein der Rezipient des Romans als Element des realen Raums übrigbleibt.

Der Text führt durch re-entrys, die Realität/Virtualität-Differenz immer wieder neu ins System der Fiktionalität ein, bis das letzte re-entry einerseits die Differenz auf Rezipienten-Text-Ebene manifestiert, aufgrund der plötzlichen Auflösung mit dem letzten Satz das Bewusstsein des Rezipienten nachhaltig erschüttert. Somit wird, ähnlich wie bei Goetz die Realität/Fiktionalitäts-Differenz als Einheit markiert. Dies gelingt, weil sich Der Kameramörder klassischen Inszenierungsstrategien filmischer Räume bedient und der Rezipient ähnlich wie beim Film den diegetischen Raum über die gezeigten Orte hinaus auf eine spezifische Weise verlängert, ohne jedoch die Beobachtungsebenen als Einheit zu sehen ${ }^{17}$.

17. - Khouloki über die heuristische Definition des Begriffs ,Filmischer Raum“: „Obwohl der Film nur eine Aneinanderreihung von Fragmenten ist, kann er beim $\mathrm{Zu}$ schauer die Illusion eines homogenen Raums hervorrufen. Das ist nur möglich, weil der Off-Raum, also der Teil des Raums, der nicht im Bild gezeigt wird, als Verlängerung des Bildraums selbstverständlich vorausgesetzt wird. Er spielt eine fundamentale Rolle in der Filmwahrnehmung. Der nicht im Bild präsente Teil des Raums zwischen den Einstellungen wird vom Zuschauer ergänzt, sodass jeder Einstellungswechsel innerhalb einer Sequenz in der Regel, solange nichts Eindeutiges dagegen spricht, als Sprung in 
Eine Differenzierung zwischen der Realität des Erzählers und der Realität der Fernsehbilder findet zuvor permanent statt, wenn beispielsweise der „Blick aus dem Fenster“ (KM, S. 153) dem Fernsehbild entgegengesetzt wird, bis die Beobachter selbst beobachtet werden: „Heinrich wandte sich vom Fenster ab. Er verkündete, wir seien im Fernsehen, allerhand. Er lief ins Zimmer und startete den Videorecorder, um die Sendung aufzuzeichnen.“ (KM, S. 154) Erst die Einheit dieser Differenz sorgt im letzten Absatz für die Auflösung des Kriminalfalls:

Ich sah im Fernsehen, daß Handschellen gezückt wurden, und wandte mich um. Der kommandierende Polizist erklärte mich für verhaftet. Ich sei beschuldigt, 2 Kinder ermordet zu haben. Ich leugne nicht. (KM, S. 156)

\section{Thomas Glavinic: Die Arbeit der Nacht}

Vor dem Hintergrund, dass die literarische Inszenierung filmischer Räume geeignet ist, gängige Beobachtungskonventionen zu hintertreiben, veröffentlicht Glavinic 2006 seinen dystopischen Roman Die Arbeit der Nacht ${ }^{18}$, der die filmischen Raumillusionierungen aus Der Kameramörder auf hypertrophische Weise instrumentalisiert.

Protagonist Jonas wacht eines Morgens in seiner Heimat Wien auf und stellt fest, dass alle Menschen über Nacht verschwunden sind. Er ist allein. Mit einem Truck begibt er sich auf Expedition, ,in eine leere, fremdgewordene Welt" (AN, S. 4), um das Rätsel aufzulösen. Am Ende wird er im Zustand vollständiger Depersonalisation sein Schicksal als eine von vielen Seins-Möglichkeiten akzeptieren und sich in eine glücklich-naive Kindheit fantasieren.

Birgit Holzner ${ }^{19}$ untersucht das ihrer Ansicht nach (post-)apokalytische Geschehen ${ }^{20}$ vor dem Hintergrund gesellschaftlicher Ängste, die sie als Phänomen des Milleniumswechsels qualifiziert. Jonas' Reise erscheint bei ihr als ,Spurensuche in die Vergangenheit seiner Familie“ (AN, S. 217). Julia Schöll konstatiert entgegengesetzt:

ein- und demselben Raum wahrgenommen wird." Khouloki, 2009, S. 11.

18. - Thomas Glavinic: Die Arbeit der Nacht, München, hanser, 2006. Nachfolgend zitiert als AN.

19. - Vgl. Birgit Holzner: Thomas Glavinics Endzeitroman DIE ARBEIT DER NACHT. In: Susanne Krones, Evi Zemanek (Hgg.): Literatur der Jahrtausendwende, 2008 , S. 215.

20. - Harrison schreibt in seiner Rezension für den „The Guardian“ in Zusammenhang mit Glavinics Roman über ,the post-disaster story“ und stellt bereits mit der Überschrift „I am legend“ einen direkten Zusammenhang zum tatsächlich post-apokalyptischen Science-Fiction-Film „I Am Legend“ (2007) her, obschon in Die Arbeit der Nacht an keiner Stelle die behauptete Apokalypse verifiziert wird. John M. Harrison: ,I am Legend“. In: The Guardian. 26.7.2008. 
Keine Katastrophe hat stattgefunden, kein atomarer Gau die Menschheit ausgelöscht; alle außer Jonas sind schlicht verschwunden, und der Roman folgt nun der Frage, was geschieht, wenn das Subjekt endgültig und absolut mit sich allein, somit der Andere endgültig und absolut abwesend ist ${ }^{21}$.

Die filmtopographischen Motive erwähnt Holzner kurz, wenn sie von Jonas” „Arbeit der Nacht“ erzählt. Denn der Held beginnt, sich selbst mit einer Videokamera aufzunehmen, und führt im Schlaf als „,Schläfer', ein beklemmendes Doppelleben, in dem er sich selbst auf sehr verstörende Weise gegenüber tritt" ${ }^{\prime 22}$.

Dabei eröffnet der Roman bereits mit medial vermittelten Räumen, wenn „der Bildschirm flimmert[e]“ (AN, S. 7) und Jonas beim Durchschalten der Fernsehprogramme „kein[en] Empfang“ (Ebd.) erhält: „Er schaltete den Fernseher ein. Flimmern. Er schaltete den Computer ein. Server error. Er schaltete das Radio ein. Rauschen.“ (AN, S. 9). Die medialen Räume sind leer, ebenso die Straßen, Brücken, Plätze (die Außenräume) (AN, S. 10), die Büros (AN, S. 11) und die Privaträume seines Vaters (AN, S. 14) (die Innenräume).

Khouloki beschreibt anhand von Stanley Kubricks 2001: A Space Odyssee den Aufbau sowohl agoraphobischer als auch leerer Räume und schreibt über die Eingangssequenz: „Egal, wohin man schaut, überall herrscht dieselbe Leere" 23 . Zur genaueren Analyse zitiert Khouloki Gilles Deleuzes' „Unterscheidung zwischen leeren Räumen und Stilleben“ (Ebd. S. 140):

Ein leerer Raum gewinnt seine Bedeutung vor allem aus der Abwesenheit eines möglichen Inhalts, während das Stilleben sich durch die Anwesenheit und Zusammenstellung von Gegenständen definiert, die sich in sich selbst hüllen oder so zu ihrem eigenen Behältnis werden ${ }^{24}$.

„Viele Erklärungen gab es nicht.“ (AN, S. 16). In Die Arbeit der Nacht vermutet Jonas zu träumen, oder wahnsinnig zu sein. (AN, S. 17). Als alle Versuche, den realen Raum als Wirklichkeit einzunehmen, gescheitert sind, beschließt er, das Heil im Fiktionalen zu finden:

In seiner Videosammlung suchte er nach Filmen, die er noch nicht oder lange nicht mehr gesehen hatte. Er baute einen Stapel Komödien vor dem Fernseher auf. Die Jalousien ließ er heruntergefahren. (AN, S. 17)

21. - Julia Schöll: Entwürfe des auktorialen Subjekts im 21. Jahrhundert. Daniel Kehlmann und Thomas Glavinic. In: Johanna Bohley, Julia Schöll (Hgg.): Das erste Jahrzehnt: Narrative und Poetiken des 21. Jahrhunderts, 2011, S. 281.

22. - Holzner, 2008, S. 219.

23. - Khouloki 2009, S. $139 \mathrm{ff}$.

24. - Gilles Deleuze: Das Zeit-Bild. Kino 2. Frankfurt, Suhrkamp, 1997, S. 30. 
Nach einigen Tagen installiert Jonas Kameras: auf dem MilleniumTower, (AN, S. 74) am Stephansdom. (AN, S. 75) „Weitere Kameras stellte er vor dem Parlament auf, vor der Hofburg, im Burgtheater, auf der Reichsbrücke, in einer Straße im Bezirk Favoriten." (AN, S. 78). Auf seiner Reise wird er später die gesamte leere Landschaft mit Kameras beobachten. „Mal meinte er, die Öde nehme kein Ende, er sah grau in grau, verfallene Schuppen, verbrannte Felder, häßliche Fabriken, Kraftwerke. Ihm war alles einerlei. Mit präzisen, immergleichen Handbewegungen postierte er seine Kameras und stieg wieder in den Lkw.“ (AN, S. 316). Bereits ab Seite 100 nimmt er sich während des Schlafs auf.

Die im Text beobachtete Figur wird selbst zum Beobachter und verdoppelt ihre Realität in der Hoffnung, Bewegungen aufzunehmen. Doch wenn Jonas die Videobänder betrachtet, wird eine unheimliche Differenz zwischen Realität und Fiktion offenbar. Die auf dem Bildschirm gezeigte Fläche steht, ebenso wie in den oben angeführten Filmen der 2000er Jahre in keinem mimetischen Verhältnis zur Realität.

Mal ist das Band leer, obschon er ,alle - alle! - nötigen Knöpfe gedrückt" (AN, S. 89) hat, dann variiert das Bild bei mehrmaligem Ansehen (AN, S. 104). Jonas differenziert sein beobachtendes Dasein am Tag und der Beobachtung seiner Selbst in der Nacht. Betrachtet er die Videobilder seines Schlafs, so verfolgt er „ein Geschehen, das er nicht verstand“, (AN, S. 130) der Schläfer trägt an einer Stelle eine maskenartige Kapuze: „Jonas konnte nicht lange in diese Maske schauen. Er meinte in ein Loch zu blicken, seine Augen ertrugen die Leere nicht", (AN, S. 173) er ist sich sicher: „Ich bin es nicht, der Schläfer!“ (AN, S. 296). Wenn dieser auf dem Bild zwinkert, ist sich Jonas sicher, in der vergangenen Nacht „keinesfalls in die Kamera gezwinkert“ (AN, S. 301) zu haben.

Zwischen Realität und Beobachtung wird in Die Arbeit der Nacht eine Differenz behauptet, die überhaupt erst glaubwürdig wird aufgrund des kulturell vermittelten Wissens um die neue räumliche Illusionierung im Film. Erst nachdem im cineastischen System eine Differenz zwischen diegetischer Realität und rezeptionsästhetischer Beobachtung eingeführt wurde, funktioniert die Behauptung dieser Differenz auch auf dem literarischen Feld, wie in Der Kameramörder oder Die Arbeit der Nacht.

\section{Thomas von Steinaecker: Geister}

Der nur ein Jahr nach ,Wallner beginnt zu fliegen“ erscheinende zweite Roman Geister (2008) führt viele Themen des Debüts fort. Auch hier spielen gescheiterte Lebensläufe. Gedankenspiele, Tagträume und 
Parallelwelten, aber auch die verschiedensten medialen Wirklichkeiten aus Film, Fernsehen, Fotografie und Comics eine wichtige Rolle ${ }^{25}$.

Der Roman beginnt, zunächst unmarkiert, als Film im Text. ,Jürgen sieht seiner Geburt zu. Das Gesicht seiner Mutter ist schmerzverzerrt" 26. Es folgt auf der gleichen Druckseite die Wiedergabe des Abspanns, „Regie, Produktion, Wir danken der Familie Kämmerer“ (G, S. 7) und wenige Absätze später eine Ansprache des Regisseurs Werner Bischoff 27 :

Ja, noch ein Wort zu den Kameras, bitte nicht erschrecken. Das hat nämlich folgende Bewandtnis: Ich drehe gerade einen neuen Film über die Familie Kämmerer, das Sequel sozusagen [...] Ich bitte jetzt also Frau und Herrn Kämmerer auf die Bühne. (G, S. 8)

Der Roman verhandelt die Traumabewältigung einer Familie. Bereits vor Jürgens Geburt verschwindet seine Schwester Ulrike spurlos. Es wird vermutet, sie sei ermordet worden. Ihre Leiche wurde nie gefunden. Besagter Regisseur begleitet die Suche der Eltern ebenso wie das Leben mit der nur medial vermittelten Leerstelle ${ }^{28}$ Ulrike bei Jürgen selbst.

Die Realitätsverdopplung, die Subjekt und Objekt des Erzählens zum Ende des Romans Der Kameramörder und mittig von Die Arbeit der Nacht in eins setzt, wird hier bereits am Anfang markiert. Zugleich erscheint der literarische Text in Differenz zum filmischen Raum, das sprachliche Erzählen wird als ein anderes Bereitstellen von Informationen inszeniert, wenn Jürgen Kämmerer beispielsweise bemerkt:

Und dann hat Ulrike tatsächlich da gestanden. Für den Bruchteil einer Sekunde hat Jürgen sie für eine Sinnestäuschung gehalten, seine Schwester, in der rechten Hand den Teddybären, in der linken die Gießkanne, rote Gummistiefel, Zahnlückengrinsen, genau wie auf dem Foto, dem Lieblingsfoto der Eltern, nein, es ist das Foto, das leicht verschwommen eingeblendet wurde und über das nun der Abspann läuft, Regie, Produktion, Wir danken der Familie Kämmerer. (G, S. 7)

25. - Wolfgang Reichmann: „Thomas von Steinaecker,“ In: Hermann Korte: Kritisches Lexikon zur deutschsprachigen Gegenwartsliteratur. München. text + kritik., S. 3 (Stand: 15.06.2011).

26. - Thomas von Steinaecker: Geister, Frankfurt, FVA, 2008, S. 7. Nachfolgend zitiert als: $\mathrm{G}$.

27. - Der Name Bischoff kann als Hinweis auf die geradezu theologische Erzählerautorität des Regisseurs gelesen werden.

28. - Was Jürgen über Ulrike weiß, erfährt er aus nachgestellten Dokuspielszenen, in denen Ulrike von einer kindlichen Darstellerin verkörpert wird (G, S. 12), vermittels Fotografien, die ,sich überall im Haus befinden (G, S. 14), aus Erzählungen seiner Eltern (G, S. 28). 
Entscheidend ist, in welcher Weise filmische Rauminszenierungen auf die intern sehr nah fokalisierte Erzählung von Jürgen einwirken. Er übernimmt direkt technische Verfahren zur Raumkonstruktion, wenn er plötzlich ,einen auf Schnelldurchlauf [macht]“, (G, S. 19) sein Dasein mit einer Zeitschleife vergleicht, ,wie in einem dieser Science-FictionFilme“, (G, S. 16) bereits in seiner Realität aus dem Film bekannte Geigen-Untermalungen hört, (Vgl. G, S. 66) wenn er in Bezug auf seine Großmutter bemerkt, sie sei ,ein Fernseher, es gibt drei Programme [...] Drittes Reich, Nachkriegszeit, Alltag“", (G, S. 39) wenn er filmisch vermittelte Inszenierungen als Konstruktionen entlarvt: „Jürgen ist sich fremd. Der, den er da auf dem Bildschirm sieht, könnte auch ein anderer als er selbst sein." (G, S. 72). Dabei wird sein Lebensraum durch die filmische Erzählung nicht nur ontologisch beeinflusst: „Auf der Rückfahrt nach Hause sind sie mit dabei und drehen, der Kameramann hinten zwischen dem Regiefuzzi und Jürgen, für den kaum noch Platz ist" (G, S. 12).

Hinzu kommen die aktualisierten Virtualitätsebenen, bei Jürgens Luftgitarrensolo „mit dem imaginären Plektrum“ (G, S. 35), oder auch bei einer auf Text- und verschiedenen Bildebenen wiedergegebenen Comicreihe, die das potentielle Leben von Jürgens' Schwester fortschreibt und durch eine Dokumentation über Ulrike motiviert worden ist.

Er verwandelt und medialisiert sich. Er möchte immer zur nächsten Ebene kommen und befindet sich in dem Irrglauben, dass die nächste Ebene bereits die letzte Ebene ist. Dort würde er seine verschwundene Schwester Ulrike wiedertreffen. Zuerst meint er, der Dokumentarfilm würde ihn von seinem Trauma heilen. Dann zehn Jahre später die Comics. Aber auch das ist nicht des Rätsels Lösung. Weil da eben Cordula Maas ins Spiel kommt, eine verquaste Liebe und Beziehung. Die Ebenen geraten durcheinander. (Vgl. G, S. 125)

Entscheidend in Bezug auf die anfängliche These meines Aufsatzes ist, dass Geister in den Rezeptionsraum transformiertes kulturelles Wissen um Konstruktion, Wahrnehmung und Bedeutung filmischer Räume aktualisiert, um die immer wieder neue Realität-Fiktion-Differenz explizit zu machen. Das Verhältnis der filmischen „Dokumentation“ zur Realität erscheint hier ebenso arbiträr wie System-Beobachtungs-Beziehungen innerhalb der Text-Diegesis. Filmische Topographien werden in Geister instrumentalisiert, um obige Differenz auf mannigfaltige Weise zu exemplifizieren ${ }^{29}$, zudem können auf intertextueller Ebene Verweise auf Filme

29. - Elmar Krekeler nennt Geister den „wahrscheinlich ersten medialvernetzten Roman der jüngeren deutschsprachigen Literatur. [...] Tom Tykwers Lola rennt regelmäßig an Menschen vorbei, deren mögliches zukünftiges Schicksal immer wieder anders im Zeitraffer erzählt wird, und verwandelt sich regelmäßig in eine Comic-Figur. Was auch Lolas Fernsehschwester tut, die Comic-Zeichnerin Lolle in der Serie ,Berlin 
wie Die Freunde der Freunde ${ }^{30}$ von Dominik Graf oder Gespenster von Christian Petzold festgestellt werden.

\section{Ausblick und Schluss}

Musste Rainald Goetz für die Darstellung dieser Differenz noch echtes Blut auf das Papier tropfen lassen, diskutieren die Texte Glavinics und von Steinaeckers besagte Differenz auf zeittypische Weise mit Rauminszenierungen, die in den 2000er Jahren verstärkt über neue filmische Beobachtungsverfahren in den kulturellen Raum überführt worden sind. Wissenschaftliche Analysen von Literatur jener Zeit, die sich auseinandersetzen mit rhizomatischem Schreiben und Lesen ${ }^{31}$, novellistischen Erzählmodellen ${ }^{32}$, Narrativen der New Economy ${ }^{33}$ oder auch Form und Funktion historisch-fiktionalen Erzählens ${ }^{34}$ gibt es ebenso, wie jene über Ästhetiken totaler Medialisierung in internationaler Literatur ${ }^{35}$ usw.

Allein wie neue Filmtopographien im Sinne Khoulokis die Konstruktion, Wahrnehmung und Bedeutung literarischer Texte verändert haben, ist bislang unbeobachtet geblieben. In der Analyse wurde nun belegt, in welcher Weise eine Neuinszenierung filmischer Räume mit daran anschließenden literarischen Verfahren korreliert. Glavinic hat als einer der ersten Autoren deutschsprachiger Gegenwartsliteratur neue Rauminszenierungen aus dem Film auf Texte übertragen und insbesondere in den Romanen Der Kameramörder und Die Arbeit der Nacht

Berlin`. Nebenbei feiert auch die legendäre und unglaublich wahre Flitcraft-Episode von Dashiell Hammett fröhliche Urständ, die uns schon in Paul Austers ,Nacht des Orakels“ und jüngst Judith Kuckarts ,Die Verdächtige“ begegnete.“ Elmar Krekeler: „Das Ich ist nicht genug." In: Die Welt, 8.11.2008.

30. - „Das Buch funktioniert als ständige mediale Transformation. Es gibt Subtexte und Prätexte, die ich verarbeite. So wie der Text selbst eine ständige mediale Verarbeitung ist. Zum Beispiel waren zwei Filme wichtig für Geister. Das waren Die Freunde der Freunde von Dominik Graf, eine Internatsgeschichte und Gespenster von Christian Petzold eine Entführungsgeschichte, die aus einer völlig anderen Perpektive erzählt wird als das Drama um Ulrike Kämmerer in Geister. “ www.zeit.de/online/2008/37/ interview-steinaecker/seite-3.

31. - Sabine Zubarik: Rhizomatisches Schreiben (und Lesen): Albert Goldbarths PIECE OF PAIN, In: Susanne Krones/Evi Zemanek, 2008, S. $317 \mathrm{ff}$.

32. - Andreas Blödorn: Restaurative Konsolidierung - oder Wiederkehr und Aufschub verdrängter Krisen. Beobachtungen zur Abstraktion eines novellistischen Erzählmodells der 2000er Jahre. In: Bohley/Schöll, 2012, S. 37 ff.

33. - Anke Biendarra: Prekäre neue Arbeitswelt: Narrative der New Economy. In: Bohley/Schöll, 2012, S. 69 ff.

34. - Stephanie Catani: Was bleibt von der Geschichte? Form und Funktion historisch-fiktionalen Erzählens im 21. Jahrhundert. In: Bohley/Schöll, 2012, S. 23 ff.

35. - Karin Peters: „Todo se mantrifica“ oder: Die absolute Telenovele. Rodrigo Fresán und die Ästhetik totaler Medialisierung in ,Mantra'. In: Susanne Krones, Evi Zemanek, 2008, S. 69 ff. 
mit gestörten Realität/Fiktion-Differenzen operiert. Dokumentarische Bilder erscheinen bei Glavinic, aber auch in von Steinaeckers Geister als inkonsistent und in einem arbiträren Verhältnis zur Realität gesetzt.

Dieses Verfahren kann seit den 2000er Jahren mannigfaltig in der deutschsprachigen Gegenwartsliteratur beobachtet werden. Es prägt als ein anti-mimetisches Konzept Texte bis in die jüngste Gegenwart. Wolfgang Herrndorfs Erzählung Diesseits des Van-Allen-Gürtels zeichnet die Analyse von Filmbildern der ersten Mondlandung nach. Vermittels rauminszenatorischer, aus Hollywood bekannter Verfahren wird hier ein Misstrauen gegenüber dokumentarischen Narrationen manifestiert ${ }^{36}$. In Schimmernder Dunst über CobyCounty von Leif Randt stehen filmdokumentarische Rauminszenierungen in einem ambivalenten Verhältnis zu einer ohnehin nur noch medial vermittelten Realität ${ }^{37}$. Figuren „bewegen sich so, als würden sie sich alle zeitgleich an alte Camcorderaufnahmen von ihren früheren Tänzen im Regen erinnern" 38 . Später schauen sich die in CobyCounty lebenden Figuren ,eine leicht farbkorrigierte Langversion von ,Schimmernder Dunst über CobyCounty “" an, während die ganze Zeit unklar bleibt, „ob es den Ort jenseits dieser total(itär) gewordenen Utopie bzw. Dystopie CobyCounty überhaupt gibt“"39.

Somit erscheint offensichtlich, dass mithilfe neuerer Formen filmischer Raumgestaltung eine Differenz markiert wird, die zwar nicht klärt, ob Literatur als Teil der Realität, oder als Beobachtung 2. Ordnung qualifiziert werden kann, die anfängliche, nach Luhmann gestellte Frage jedoch auflöst in einen mehrdeutigen Raum „besserer Differenzen“40.

Dies hat nichts mit Science-Fiction oder Phantastik zu tun; vielmehr handelt es sich um das nie realisierte, um Projekte und Träume, die Nachtseite der Welt der Fakten [...], die letztlich genauso viel über die Gesellschaft auszusagen vermag wie das tatsächlich Geschehene ${ }^{41}$.

36. - Wolfgang Herrndorf: Diesseits des Van-Allen-Gürtels, Berlin, Eichborn, 2007, S. $149 \mathrm{ff}$.

37. - Jan Drees: Die Anti-Paradiso-Hölle. Wuppertal. www.lesenmitlinks.de (17.08.2011).

38. - Leif Randt: Schimmernder Dunst über CobyCounty. Berlin. Bloomsbury, 2011, S. 11.

39. - Moritz Baßler: Schimmernder Dunst - Konsumrealismus und die paralogischen Pop-Potenziale. In: Pop. Kultur \& Kritik, Heft 1/2012, Bielefeld, transcript, S. 62.

40. - Vgl. Dirk Baecker, Georg Stanitzek (Hgg.): Archimedes und wir, Berlin, Merve, 1987, S. 127.

41. - Steinaecker, 2010, zit. n. Reichmann, S. 5. 